\title{
LAS AULAS INVERTIDAS: UNA ESTRATEGIA PARA ENSEÑAR Y OTRA FORMA DE APRENDER FÍSICA ${ }^{1}$
}

\author{
FLIPPED CLASSROOM: A STRATEGY TO TEACH AND ANOTHER WAY TO LEARN PHYSICS \\ AS SALAS INVESTIDAS: UMA ESTRATÉGIA PARA ENSINAR E OUTRA FORMA DE APRENDER \\ FISICA \\ Blanca Flor Mora Ramírez², César Augusto Hernández Suárez ${ }^{3}$
}

Fecha de recibido: Febrero 05 de 2017 | Fecha de aprobado: Abril 17 de 2017

\begin{abstract}
Resumen
La implementación de un aula invertida como estrategia para gestionar el aprendizaje de la Física en estudiantes del Colegio Manuel Antonio Rueda Jara, buscó mejorar los desempeños académicos de la asignatura, apoyando el desarrollo de competencias a través de medios tecnológicos que facilitaron momentos pedagógicos presenciales y virtuales, donde se pudo ampliar los tiempos de estudio y los canales de comunicación; considerando que invertir la clase implica repasar contenidos declarativos de bajo nivel en casa; y que la práctica, "que implica alto nivel cognitivo", se desarrollará en el aula con la asesoría del maestro. El estudio realizado tuvo un enfoque cuantitativo, de tipo descriptivo; metodología que permitió concluir que el $86 \%$ de los estudiantes encuestados se mostraron satisfechos con la estrategia; además, se mejoró el rendimiento académico de la asignatura en un $15 \%$ para los grados décimos y un $17 \%$ para los grados undécimos, de un periodo a otro.
\end{abstract}

Palabras clave: aula invertida, aula inversa, flipped classroom.

1 Artículo de investigación.

2 Licenciada en Educación Informática. Docente del Colegio Manuel Antonio Rueda Jara, Municipio de Villa del Rosario. Correo electrónico: florecita_b@hotmail.com

3 Doctor en Ingeniería de Sistemas y Computacion UNAL. Universidad francisco de Paula Santander. Correo electrónico: cesaraugusto@ufps.edu.co 


\section{Abstract}

The implementation of flipped classroom as a strategy to manage the learning of physics in students of Manuel Antonio Rueda Jara College, sought to improve the academic performance of the subject, supporting the development of skills through technological means that guaranteed online and virtual teaching spaces, where it was possible to extend study hours and communication channels; and considering that flipping the class involves reviewing low-level declarative content at home and that the practice, "involving high cognitive level", will be developed in the classroom with the teacher's advice. The study carried out had a quantitative approach of descriptive type, methodology that allowed to conclude that $86 \%$ of the students surveyed were satisfied with the strategy. In addition, the academic performance of the subject was improved by $15 \%$ for grades tenth and $17 \%$ for eleventh graders, from one period to the other.

Key Words: inverted classroom, reversed classroom, flipped classroom.

\section{Resumo}

A implementação de uma sala investida como estratégia para gerenciar a aprendizagem da Física em estudantes do Colégio Manuel Antonio Roda Jara, procurou melhorar os desempenhos acadêmicos da matéria, apoiando o desenvolvimento de concorrências através de meios tecnológicos que facilitaram momentos pedagógicos presenciais e virtuais, onde pôde ser ampliado os tempos de estudo e os canais de comunicação; considerando que investir a classe implica repassar conteúdos declarativos de baixo nível em casa; e que a prática, «que implica alto nível cognitivo», se desenvolverá no sala com a assessoria do maestro. 0 estudo realizado teve um enfoque quantitativo, de tipo descritivo; metodologia que permitiu concluir que o $86 \%$ dos estudantes interrogados se mostraram satisfeitos com a estratégia; além disso, melhorou-se o rendimento acadêmico da matéria num 15 \% para os graus décimos e um 17 \% para os graus onces, dum período a outro.

Palavras-chave: sala investida, sala inversa, fliped classroom. 


\section{INTRODUCCIÓN}

García, Pierce y Quezada sostienen que:

La mayoría de los estudiantes que ingresan a la Universidad, a estudiar carreras de Ingeniería, en el nivel introductorio presentan dificultades para desarrollar un entendimiento funcional de los conceptos básicos de la Física, dejando como resultado una deserción aproximada del $50 \%$ en la carrera de ingeniería (2008, p. 19).

La anterior estadística es preocupante, pues es claro que los estudiantes no tienen las competencias mínimas para resolver situaciones de la asignatura; las razones se evidencian en las aulas del entorno educativo oficial, donde, a la par de atender un gran número de estudiantes por salón, no se cuenta con los implementos suficientes para la realización de experiencias que faciliten la comprensión y aprendizaje de contenidos. Otro factor que influye es la metodología y la didáctica aplicada por los docentes, donde aún se ofrece la instrucción tradicional, lo que aumenta la complejidad y las falencias del aula regular; en la que, además de desatenderse los diferentes ritmos y estilos de aprendizaje, se lucha con los diferentes vacíos que tienen los estudiantes frente a una temática. Los anteriores factores inciden directamente en el alto porcentaje de pérdida escolar en materias como Matemáticas, Física y Química en la educación básica.

Ubicando la situación en la Institución Educativa y teniendo en cuenta lo evidenciado en actas de compromiso académico y planes de nivelación periódica, la asignatura de Física presenta un significativo porcentaje de desaprobación: $45 \%$, lo que demuestra que la Física es considerada por los estudiantes como una asignatura compleja, como lo manifiesta mi compañero, investigador del proyecto y docente de la asignatura Física, William Mejía Torres:

La asignatura se muestra difícil, porque los estudiantes no desarrollaron las habilidades básicas en el área de Matemáticas en los grados anteriores, las cuales se requieren para realizar operaciones matemáticas, para comprender y extraer datos relevantes de texto, para contextualizar los conceptos, lo que genera un clima tenso al mencionar la asignatura; situación que preocupa pues la intensidad horaria es poca para nivelar y dar solución a las inquietudes particulares y grupales que presentan los estudiantes. (Febrero 2016, comunicación personal).
Aprovechando la «empatía» que existía entre las TIC y los estudiantes, se pretendió extender el aula presencial con un aula virtual que apoyara la gestión del aprendizaje e implementara como estrategia invertir la clase, para que, en horarios presenciales, se trabajaran experiencias relacionadas con el hacer y el aplicar, y en casa se estudiaran los conceptos relacionados con la temática. Con esta metodología se consiguió dinamizar y optimizar los procesos de enseñanza-aprendizaje de la asignatura de Física e iniciar al estudiante en una cultura de estudio a través de medios digitales.

El presente artículo presenta los referentes teóricos que orientaron la estrategia para invertir los momentos pedagógicos y expone la metodología e instrumentos usados para medir los alcances de la investigación; asimismo, se muestran gráficos sobre los análisis y se aportan las conclusiones de la experiencia de aula invertida.

\section{REFERENTES TEÓRICOS}

\section{Aula invertida o modelo invertido de aprendizaje}

Martínez-Olvera, Esquivel-Gámez y Martínez Castillo (s.f.) en su capítulo Aula invertida o modelo invertido de aprendizaje: origen, sustento e implicaciones comparten una visión general del enfoque pedagógico del aprendizaje invertido.

El aula invertida busca invertir los momentos y roles de la enseñanza tradicional, para que la clase, habitualmente dictada por el profesor, pueda ser dada y consultada por el estudiante mediante herramientas multimedia, de manera que las clases presenciales se conviertan en exploración y práctica de los conocimientos y competencias que se requieren desarrollar. Este modelo de aprendizaje implementa diferentes métodos de trabajo interactivo, el trabajo colaborativo, la teoría del conexionismo, el constructivismo, el aprendizaje basado en problemas y la realización de proyectos.

El término aula invertida, originalmente acuñado por Lage, Platt y Treglia (2000) como Inverted Classroom - IC- fue usado para detallar la estrategia de clase implementada en una asignatura específica -Economía-. La diferencia propuesta en el aula 
invertida es el uso de tecnología multimedia - videoconferencias, presentaciones- para acceder al material de apoyo fuera del aula, estrategia mediada por tecnología. En 2012, dicha estrategia fue popularizada por Bergmann y Sams, y la denominaron Flipped Classroomm - $\mathrm{FCM}$ - o aula volteada, término más reconocido en el nivel educativo básico en Estados Unidos (Coufal, 2014; Talbert, 2014).

En este documento se referenciará a esa estrategia como aula invertida o aula volteada, ya que ambas acepciones son válidas. La expansión del FCM $-F l i$ pped Classroom Model - se debió a la difusión de los videos de Bergmann y Sams en la red, lo que los hizo ganar adeptos hasta formalizar una organización denominada The Flipped Learning Network. Igualmente, hacia el año 2004, Salman Khan inició un esquema de tutoría en YouTube que dio pie a Khan Academy, herramienta muy difundida para la obtención de material audiovisual (Lage et al., 2000).
La anterior estrategia ha buscado favorecer los diferentes estilos de aprendizaje que existen en el aula. Pero las propuestas que surgieron en dicho momento no fueron fundamentadas en teorías de la educación, sino que durante la implementación se realizaron ajustes sustentados en factores como abarcar los diferentes estilos de aprendizaje del estudiantado, promover un ritmo individual de avance y desarrollar habilidades de aprendizaje autodirigido (Martínez-Olvera et al., s.f.).

\section{Elementos básicos que conforman el modelo de aula invertida}

Para desarrollar la propuesta primero hay que basarse en la necesidad de emparejar los diferentes tipos de aprendizaje de los múltiples estudiantes congregados en el grupo. En la figura 1 se muestran los componentes que integran un aula invertida.

Figura 1. Componentes de un aula volteada

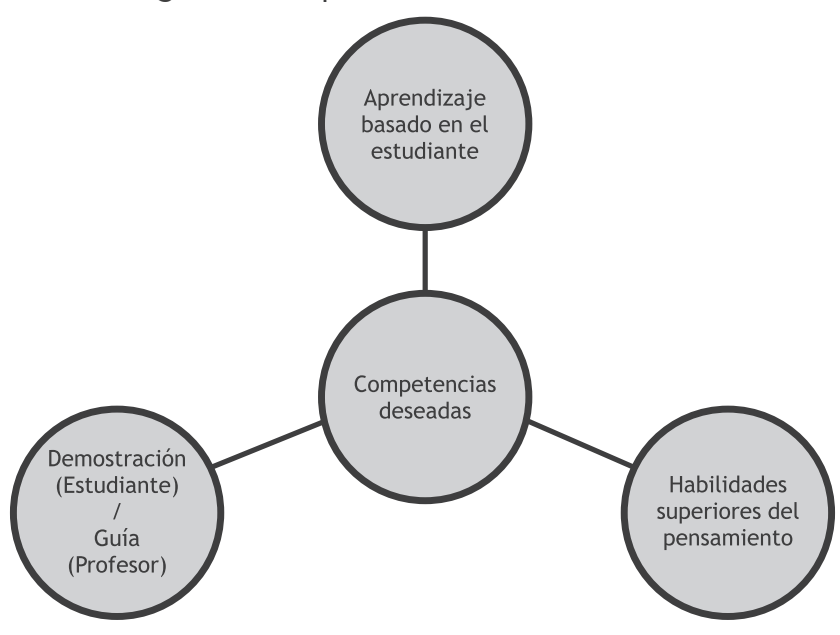

Fuente: imagen adaptada por Martínez-Olvera et al. (s.f.).

En la anterior ilustración el autor muestra como objetivo central el desarrollo de competencias y como actividad de primer orden el aprendizaje basado en el estudiante, para lo cual es menester que el docente oriente unos contenidos, y el estudiante sea capaz de demostrar buscando el desarrollo habilidades de pensamiento superior para garantizar un aprendizaje basado en el estudiante.

Invertir los quehaceres del aula -contenidos extraclase, tareas en el aula - se justifica en el hecho del repaso de contenidos declarativos, conforme a la taxonomía de Bloom, según tareas cognitivas de bajo nivel, tales como recordar y entender, en tanto que la práctica de actividades implica tareas de alto nivel como aplicar, analizar, evaluar y crear (Talbert, 2014).

Conforme el estudiante se familiarice con el uso de medios multimediales para resolver situaciones formuladas por el docente, tal estudiante podrá iniciarse en el desarrollo de habilidades de pensamiento superior o metacognición y será capaz de explorar y demostrar nuevos conceptos y conocimientos (Mazzarella \& Clemen, 2008). 


\section{Dinámica del aula invertida}

La dinámica del aula invertida sugerida por Martínez et al., es la siguiente:

Antes de iniciar cualquier sesión de clase se recomienda seleccionar el material que va a dinamizar los contenidos necesarios para el aprendizaje del estudiante. Posteriormente se da inicio a unas etapas o sesiones de implementación de contenidos en el aula presencial o virtual.

Primera sesión presencial: se inicia con un proceso de inducción y sensibilización del estudiante sobre la estrategia de las aulas invertidas, sus beneficios, metodología y criterios de evaluación, buscando motivar y pactar responsabilidades con el proyecto a ejecutar. En esta sesión se recomienda mostrar el material digital e impreso que se va a abordar durante las sesiones, los cronogramas de actividades, y organizar pequeños grupos de trabajo en clase.

Posteriores sesiones presenciales: se recomienda que la primera acción sea despejar las dudas e inquietudes de la sesión anterior. Si el tiempo de explicación excede los diez minutos, tratar dicha explicación con situaciones experimentales o de uso práctico del tema. Se sugiere, a su vez, la revisión de los cuestionarios asignados por grupos, discutir las respuestas y preparar pequeñas sustentaciones de las mismas en grupo. Ulteriormente, se propone aplicar material como cuestionarios o talleres y recolectar las evidencias para evaluar el trabajo. Para finalizar la temática, el docente nuevamente debe indagar sobre dudas e inquietudes.

Sesiones virtuales: para ampliar los tiempos de clase y dar soporte a la estrategia de aula invertida, los autores proponen la creación y uso de un sitio web donde se pueda acceder al material de trabajo y se pueda llevar el control y registro de las actividades realizadas por el estudiante en casa.

La figura que se muestra a continuación ilustra gráficamente los posibles momentos pedagógicos sincrónicos y asincrónicos que se pueden establecer.

Figura 2. Estructura del aula invertida -ilustración-

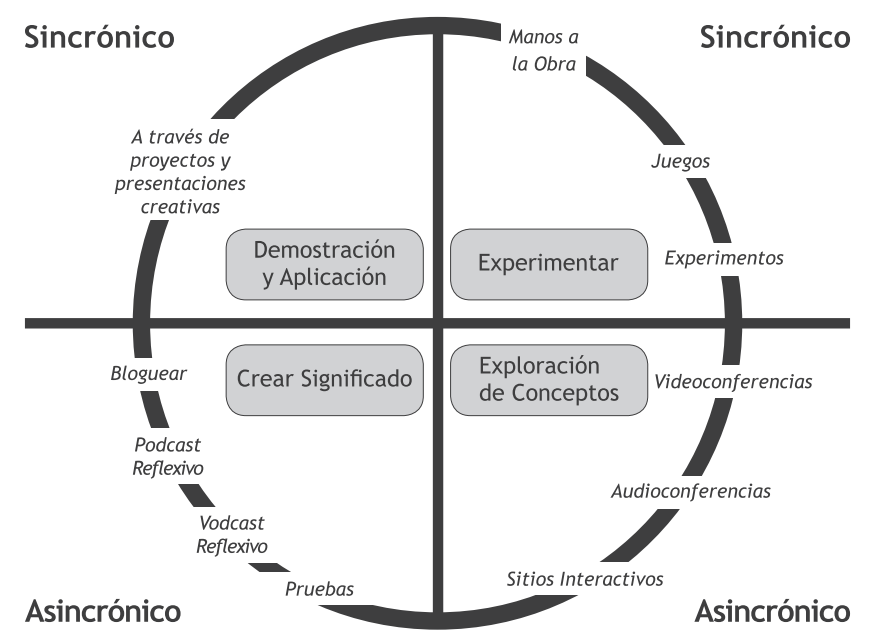

Fuente: imagen adaptada por Martínez et al. (s.f.).

\section{Dimensiones de un entorno virtual de aprendizaje}

Para lograr que un entorno virtual de aprendizaje resuelva los cuestionamientos planteados anteriormente y se logre una uniformidad didáctica y tecnológica en todas las aulas virtuales, se propone tener en cuenta los siguientes lineamientos generales de uso, planteados bajo las tres dimensiones descritas a continuación:
1. Dimensión de gestión: se relacionan todos los aspectos administrativos y de adecuación inicial que el docente debe tener en cuenta.

2. Dimensión pedagógica: establece los criterios fundamentales para la organización de todos los aspectos relacionados con los materiales de estudio y las actividades complementarias dentro de los bloques de contenido, los cuales son el corazón de un aula virtual, el sitio en donde se refleja todo el diseño didáctico, pedagógico y 
evaluativo que cada docente establece para su actividad académica con los estudiantes.

3. Dimensión evaluativa: la evaluación del aprendizaje es una actividad compleja en los procesos educativos. Con ella se busca medir y valorar el nivel de apropiación de los conocimientos y el desarrollo de procesos, habilidades y competencias de los estudiantes. Como parte del proceso educativo, se ha constituido en una oportunidad para el mejoramiento de los procesos de enseñanza-aprendizaje; así las cosas, debe ajustarse a unos patrones rigurosamente elaborados, considerando los contenidos y, acorde a estos, determinando por qué se evalúa, para qué se evalúa, qué se evalúa, cómo se evalúa y, lo más importante, a quién se evalúa. Estos aspectos permiten direccionar las estrategias de evaluación bajo la responsabilidad de los docentes (Jaramillo Mujica, 2013).

\section{Competencias científicas. ¿Qué son los} estándares básicos de competencias?

Los estándares básicos de competencias son criterios claros y públicos que permiten conocer lo que deben aprender los niños, niñas y jóvenes, y establecer un punto de referencia de lo que están en capacidad de saber y saber hacer en cada una de las áreas y niveles.

La propuesta que aquí presentamos busca crear condiciones para que nuestros estudiantes comprendan la relevancia de potenciar habilidades y construir conocimientos relacionados con las ciencias naturales, y, con estas competencias, comunicar y compartir sus experiencias, sus hallazgos, además de aportar a la construcción, cuidado y mejoramiento de su entorno natural, tal como lo hacen los científicos.

Los estándares que orienta el Ministerio de Educación Nacional -MEN- hacen parte del referente teórico y la ruta de dirección relacionadas con las competencias científicas que cada estudiante requiere desarrollar, y pretenden que:

[Los estudiantes] se acerquen al estudio de las ciencias como científicos y como investigadores, pues todo científico -grande o chico- se aproxima al conocimiento de una manera similar, partiendo de preguntas, conjeturas o hipótesis que inicialmente surgen de su curiosidad ante la observación del entorno y de su capacidad para analizar lo que observa. (MEN, 2004).
Las competencias que se enmarcan dentro de las ciencias naturales se encuentran vinculadas con las habilidades para la indagación, la explicación de fenómenos y el uso comprensivo del conocimiento científico; competencias igualmente emparentadas con un componente o entorno vivo, físico y de Ciencia, Tecnología y Sociedad -cTs-.

Competencias, indicadores de desempeño de décimos a undécimos. Competencia: procesos físicos: «Explico las fuerzas entre objetos como interacciones debidas a la carga eléctrica y a la masa».

Competencia Ciencia, Tecnología y Sociedad: «Utilizo modelos biológicos, físicos y químicos para explicar la transformación y conservación de la energía».

\section{METODOLOGÍA}

Este proyecto se desarrolló con un enfoque de carácter cuantitativo, con recolección de datos antes, durante y después de la implementación de la estrategia del aula invertida de aprendizaje, de tipo descriptivo, y midiendo de manera independiente las variables con las que se tiene relación. Según Hernandez Sampiere, Fernandez Collado y Baptista Lucio (1997), «se pueden integrar las mediciones de cada una de dichas variables para decir cómo es y cómo se manifiesta el fenómeno de interés. [...] lo descriptivo se centra en medir, con la mayor precisión posible» (p. 61). Se buscó determinar en qué medida un aula invertida, en un contexto como el nuestro, apoya el aprendizaje de estudiantes de la educación media.

El diseño metodológico es de tipo preexperimental, en el que no existe ningún control riguroso de las variables y no se constituye un grupo de control. Se hizo un diseño de pretest y postest con un solo grupo para determinar en cuanto se mejora el aprendizaje de la física, invirtiendo los momentos, apoyados en herramientas mediáticas. De acuerdo a Sánchez y Reyes, la metodología preexperimental «no reúne los requisitos de un experimento puro, por lo tanto, no tiene validez interna, pero realizan un control mínimo» (p. 70).

\section{Etapas del desarrollo del proyecto}

1. Diseño del aula invertida de aprendizaje: selección de contenidos y actividades.

2. Sensibilización de estudiantes sobre la importancia de la asignatura, la temática y la plataforma. 
3. Ejecución de actividades en el aula invertida por lecciones programadas, cada lección contenía documentos de apoyo, foros temáticos y programación de chat para resolver dudas.

4. Clase presencial: aplicación de instrumentos para validar actividades de preparación para la clase. Realización de laboratorios con materiales económicos y de fácil adquisición para explicar la aplicación de la física -circuitos eléctricos, funcionamiento de los fluidos, movimiento armónico simple, leyes de Newton-.

5. Aplicación de cuestionarios una vez terminadas las lecciones para valorar el aprendizaje de la misma; de no ser exitosa, retroalimentar en el aula las falencias presentadas.

6. Aplicación del postest para comparar el nivel de desempeño del aula en el desarrollo de competencias.

7. Tabulación y análisis de los datos bajo el programa Excel para sacar conclusiones y recomendaciones del presente proyecto.

La población objeto de estudio estuvo constituida por estudiantes de décimo y undécimo de la sede central del colegio Manuel Antonio Rueda Jara, del municipio de Villa del Rosario. Se tomó como muestra la totalidad de la población cien estudiantes, con el fin de validar específicamente cada uno de los casos teniendo en cuenta el rendimiento académico y el trabajo de aula invertida.

\section{Instrumentos}

Instrumento 1. Ficha de seguimiento a estudiantes Diseñado para control de las actividades desarrolladas por los estudiantes en el aula invertida, con registro semanal.

Instrumento 2. Ficha de estudiantes que requieren apoyo

En dicho instrumento se registraron los estudiantes que presentaron dificultades, y se les programó las actividades de apoyo. Dicho instrumento se implementó finalizando la unidad temática.

Instrumento 3. Test para evaluar el aula invertida de aprendizaje

Para evaluar la efectividad del aula invertida se diseñó un cuestionario tipo test, con 21 ítems, separados por gestiones, el cual se aplicó al finalizar la ejecución del proyecto, y pretendió evaluar la gestión del aula invertida como apoyo a la temática de la asignatura de Física.
Los instrumentos fueron cuestionarios con respuestas cerradas y abiertas. La población de estudio fueron los estudiantes de grado undécimo y décimo de la Institución Educativa Manuel Antonio Rueda Jara.

Para aplicar el test final se tomó una muestra y se ejecutó un muestreo aleatorio simple a la totalidad de la población.

\section{RESULTADOS}

El software utilizado para procesar datos fue Microsoft Excel. En tal programa, primero, se genera una matriz general de la totalidad de las respuestas por estudiante; segundo, se van agrupando dichas respuestas por gestión; y por último, se grafican los ítems agrupados para dar descripción a cada uno y responder la pregunta formulada en el problema: ¿en qué medida la implementación de un aula invertida apoya el aprendizaje de la física en los estudiantes de décimo y undécimo grado del colegio Manuel Antonio Rueda Jara?

Figura 3. Gestión administrativa

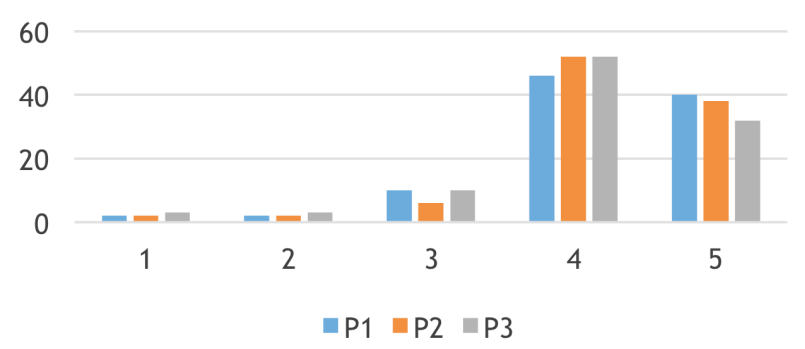

Fuente: el autor.

Los ítems 1,2 y 3 del cuestionario validan indicadores como calidad de los contenidos, herramientas de comunicación e interacción, cronograma de actividades, la programación y uso de recursos, en fin, ítems de la gestión pedagógica y administrativa en los que se obtiene que el $50 \%$ de los estudiantes seleccionaron la opción 4, y $36.7 \%$, la opción 5. Sumando estos valores, se tiene que el $86.7 \%$ de estudiantes se muestran satisfechos con la dimensión administrativa. Lo anterior demostró que la implementación y organización del aula invertida fue aprobada y apreciada por los estudiantes participantes del proyecto.

Para dar respuesta sobre qué tanto se apoyó el aprendizaje de la física, mientras se implementó el aula invertida se tuvieron en cuenta los reportes 
académicos del primer periodo versus el segundo periodo de cada uno de los grados y grupos de estudio. Considerando los reportes académicos de cada uno de los grados durante el primer y segundo periodo y comparando su rendimiento, se observa que:

Figura 4. Reporte académico grado décimo

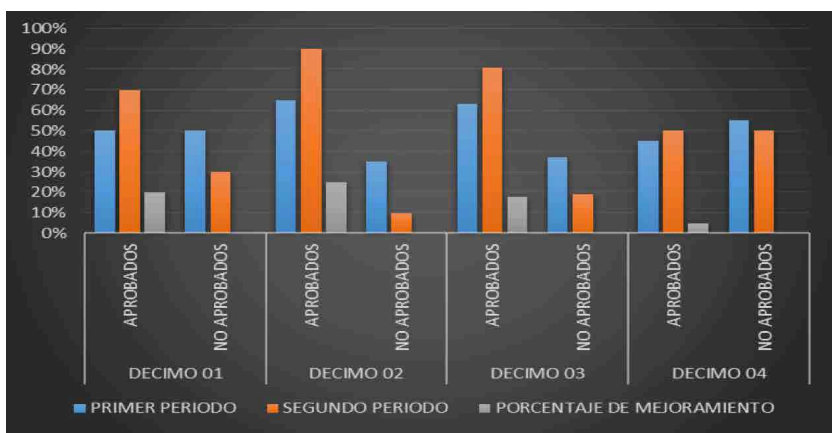

Fuente: el autor.

Para el grado décimo, el porcentaje de mejoramiento fue del $20 \%, 25 \%, 18 \%$ y $5 \%$ respectivamente, siendo décimo 04 el grado que menos mejoró su nivel de aprobados y que manifestó poco interés para participar de las actividades. Observando la ficha de seguimiento a estudiantes, se puede concluir que los grupos con mayor asistencia y trabajo en el aula invertida presentan un mejoramiento igualmente proporcional: a mayor trabajo, mejor reporte. Lo que indica que promediando los cuatro valores de mejoramiento, se obtiene un $15 \%$ de apoyo al aprendizaje y mejoramiento de la asignatura de un periodo a otro, por lo que, si se continuara la implementación en el tercer periodo, se deberían mejorar los porcentajes de aprobados por grado.

Figura 5. Reporte académico grado undécimo

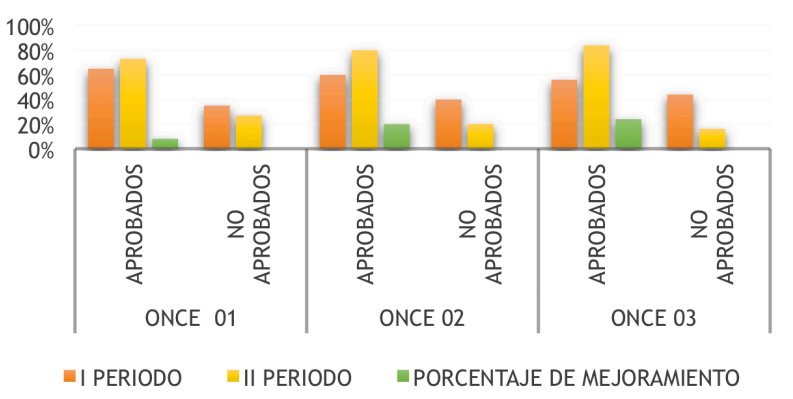

Fuente: el autor.

Para los grados once el porcentaje de mejoramiento fue del $8 \%, 20 \%$ y $24 \%$ respectivamente; promediando estos valores obtenemos que se mejoró en un $17 \%$ en el apoyo al aprendizaje y mejoramiento de la asignatura de un periodo a otro, lo que indica que si se continua con la implementación en el tercer periodo, se ha de mejorar los porcentajes de aprobados por grado.

\section{Apreciaciones sobre el proyecto}

Tomando como referente el marco teórico y la experiencia aplicada en la institución educativa, se sostiene que la estrategia de invertir los momentos de aprendizaje es una buena opción que ayuda en el desarrollo de habilidades y competencias de cualquier área. Es claro que se debe cultivar al estudiante en el uso de medios audiovisuales para aprender; además, es relevante resaltar que el aula invertida permite la discusión pedagógica, lo que fomenta en el estudiante el pensamiento crítico y el trabajo colaborativo a la hora de organizar equipos y asumir un rol en el que se fortalecen temas que a nivel individual son difusos o dudosos.

También es necesario recalcar que para implementar este tipo de proyectos se debe contar con los elementos tecnológicos requeridos para el desarrollo de las actividades inversas. En este proyecto esta fue una de las dificultades, dado que no todos los estudiantes tenían la infraestructura tecnológica pc y acceso a internet- en casa, razón que obligó a la activación de aulas en jornada contraria y a la fotocopia de material que permitiera continuar con la estrategia de manera normal. A día de hoy las instituciones educativas no cuentan con la conectividad permanente y suficiente para implementar dicha estrategia, careciendo de interactividad con el material de estudio.

Otra de las situaciones observadas es que los estudiantes poco aplican las técnicas de estudio para el desarrollo de actividades escolares, es una falencia que se evidencia en la disciplina, organización y estructuración de tareas, para lo que se hace necesario que se enseñen y exijan métodos de estudio que los ayuden a ser eficientes y efectivos en sus labores académicas.

\section{CONCLUSIONES}

Terminado el proceso de implementación del aula invertida como estrategia para apoyar el desarrollo de competencias de la asignatura de Física, podemos concluir que:

El $86.7 \%$ de los estudiantes se mostraron satisfechos con el desarrollo de la gestión administrativa, lo que prueba que el estudiante necesita que los docentes 
implementen la web 2.0 con el propósito de facilitar la comunicación e interacción a través de plataformas, redes, blogs, entre otros recursos, y con estos poder ampliar los canales de comunicación y orientación de temáticas.

En cuanto al rendimiento académico, comparando los periodos 1 y 2 , hubo, respectivamente, un mejoramiento del 15 y $17 \%$ para los grados décimo y once -en los que se redujo el nivel de no aprobados-. Se espera que con la continuidad del proyecto el porcentaje de aprobados siga mejorando hasta reducir significativamente la no aprobación en el periodo.

En los grupos analizados, un porcentaje significativo $-83 \%$ - se muestra empático con este tipo de metodología, pero también se registró que el $17 \%$ de los estudiantes se sienten molestos con la estrategia y tareas fuera del aula; todo un reto para el maestro, el cual debe sensibilizar y demostrar que con el apoyo de la tecnología se mejoran los aprendizajes.

Otro aspecto relevante es el material con que el estudiante prefiere aprender: el $80 \%$ de los estudiantes manifiestan que prefieren conceptualizar y documentarse con material multimedia, videos y libros electrónicos, de lo que se concluye que es importante que el docente organice los ejes temáticos con material interactivo para que el estudiante pueda apropiarse de los conceptos en casa y, de esta forma, aproveche los momentos presenciales para aplicar los conceptos aprendidos.

Los grupos que realizaron las actividades a cabalidad, ya sean de décimo y undécimo, mostraron mejor desempeño que los grupos que poco trabajaron en las actividades invertidas. Otro aspecto destacable es el instrumento que se implementó para el seguimiento de actividades: se probó que realizar un seguimiento minucioso a cada una de las tareas hace que el estudiante cumpla y evita el reporte de incumplimientos.

La gran mayoría de los estudiantes manifestaron su agrado con la asignatura cuando se realizaban laboratorios y expresaron que la física se aprende haciendo o simulando situaciones. La manipulación del material después de haber estudiado los conceptos reafirma el saber.

La estrategia aplicada de invertir el aula de clase es productiva e innovadora, pues posibilita la combinación de materiales, metodologías y estrategias que apoyan el desarrollo del aprendizaje en diferentes áreas del conocimiento.
Figura 1. Laboratorios de electricidad

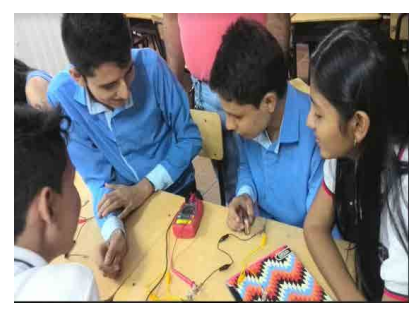

Fuente: autor del proyecto.

Figura 3. Entregando evidencias de trabajo en casa

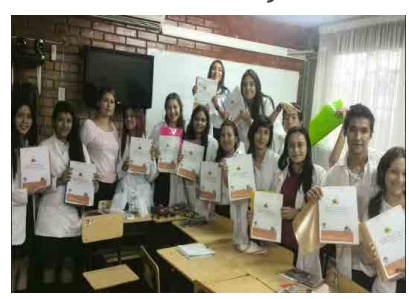

Fuente: autor del proyecto.

Figura 5. Laboratorios de electrónica

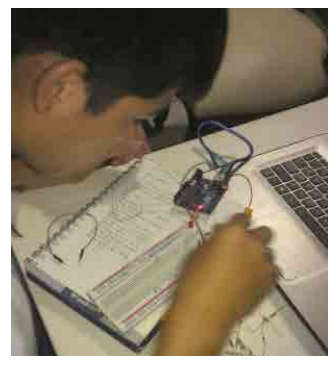

Fuente: autor del proyecto.

Figura 7. Construyendo circuitos

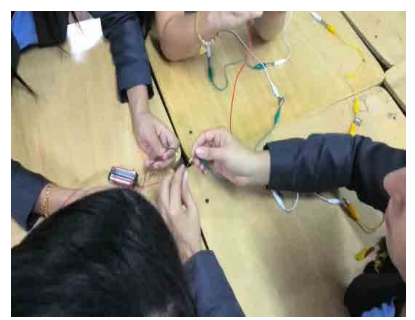

Fuente: autor del proyecto.
Figura 2. Retroalimentación de actividades

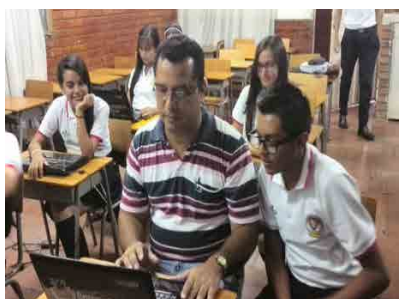

Fuente: autor del proyecto.

Figura 4. Jornada de estudio

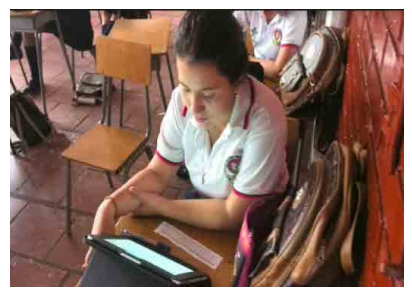

Fuente: autor del proyecto.

Figura 6. Estudiando material multimedial, retroalimentado ejercicios

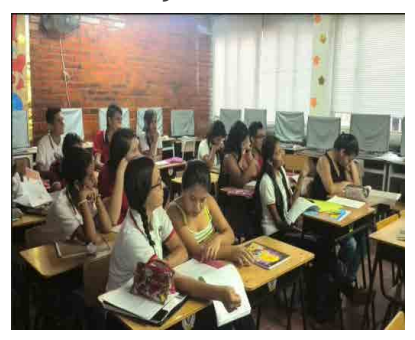

Fuente: autor del proyecto.

Figura 8. Socializando actividades

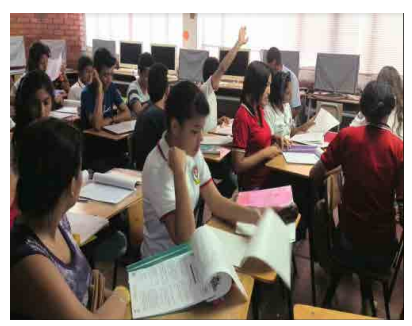

Fuente: autor del proyecto.

\section{REFERENCIAS}

Argudín, Y., \& Argudín, M. (2007). Procesos docentes I, II, III. México: Posgrado en Historiografía/ UAM-A. Recuperado de http://hadoc.azc. uam. $\mathrm{mx} / \mathrm{menu} / \mathrm{menu}$.htm

Bolivar Botia, A. (2008). Competencias básicas y ciudadanía. Caleidoscopio, revista de contenidos educativos del CEP de Jaénm,(1), 4-32. 
Clarenc, C., Castro, S., López de Lenz, C., Moreno, M., \& Tosco, N. (2013). Analizamos 19 plataformas de e-Learning: investigación colaborativa sobre LMS. Grupo GEIPITE, Congreso Virtual Mundial de e-Learning. Recuperado de www.congresoelearning.org

Flores-García, S., Chávez-Pierce, J., Luna-González, J., González Quezada, M., González-Demoss, M., \& Hernández-Palacios, A. (2008). El aprendizaje de la física y las matemáticas en contexto. Culcyt, 5(24), 19-24.

González Weil, C., Martínez Larraín, M., Martínez Galaz, C., Cuevas Solís, K., \& Muñoz Concha, L. (2009). La educación cientifica como apoyo a la movilidad social: desafíos en torno al rol del profesor secundario en la implementación de la indagación científica como enfoque pedagógico. Estudios pedagógicos, $X X X V(1)$, 63-78.

Hernández Sampiere, C., Fernandez Collado, C., \& Baptista Lucio, P. (1997). Metodología de la investigación. México: McGraw-Hill.

Jaramillo, J. (2013). Un modelo de aula virtual en Moodle con uso de herramientas Web 2.0. 1-20. Recuperado de http://www.virtualeduca.info/ponencias2013/306/modeloAulaVirtual.docx

Lage, M., Platt, Glenn., \& Treglia, M. (2000). Inverting the Classroom: A Gateway to Creating an Inclusive Learning Environment. Recuperado de https://maliahoffmann.wikispaces. $\mathrm{com} /$ file/view/inverted+classrm+1.pdf

Martínez-Olvera, W., Esquivel-Gámez, I., \& Martínez Castillo, J. (2014). Aula Invertida o Modelo Invertido de Aprendizaje: Origen, Sustento e Implicaciones. En I. Esquivel Gámez, Los Modelos Tecno-Educativos, revolucionando el aprendizaje del siglo XXI (pp. 143-160). México: Licencia Creative Commons.

Mazzarella, C. (2008). Desarrollo de habilidades metacognitivas con el uso de las TIC. Investigación y Postgrado UPEL-IPC. Recuperado de file:///D:/Nueva \%20carpeta/877-1939-1PB.pdf

Ministerio de Educación Nacional [MEN]. (2004). Formar en ciencias, jel desafío! Lo que necesitamos saber y saber hacer. Bogotá: Ministerio de Educación Nacional.

Muñoz Álvarez, G. (2010). La enseñanza de la física mediante la resolución de problemas y las relaciones Ciencia, Tecnología y Sociedad [Tesis de pregrado]. Medellín: Universidad de Antioquia.

Observatorio de Innovación Educativa del Tecnológico de Monterrey. (2014). Reporte EduTrends: aprendizaje invertido. Monterrey: Tecnológico de Monterrey. Recuperado de http://www.sitios.itesm.mx/webtools/ Zs2Ps/roie/octubre14.pdf

Talbert, R., (2012). Inverted Classrom. Recuperado de http://scholarworks.gvsu.edu/cgi/ viewcontent. cgi?article $=1183 \&$ context $=$ colleagues-

Reyes Ortíz, C. (2009). Estrategias académicas con aulas virtuales. Recuperado de https://slidedoc.es/estrategias-academicas-con-aulas-virtuales-e-learning-en-periodos-de-evaluacion-extraordinaria-pdf

Universidad Autónoma Metropolitana. (s.f.). Entornos virtuales de aprendizaje. Recuperado de http: / / sgpwe.izt.uam.mx/files/users/virtuami/file/int/practica_entornos_actv_AVA. pdf 